\title{
Original Article \\ Effects of forward head posture on static and dynamic balance control
}

\author{
Joon-Hee Lee, PT, PhD ${ }^{1)}$ \\ 1) Department of Physical Therapy, Cheongju University: 298 Daesung-ro, Sangdang-gu, Cheongju, \\ Republic of Korea
}

\begin{abstract}
Purpose] To determine the effects of forward head posture on static and dynamic balance control. [Subjects and Methods] This study included 30 participants who were included into a forward head posture group $(\mathrm{n}=14)$ and a control group $(\mathrm{n}=16)$ according to their craniovertebral angles. Static balance control was assessed according to center of gravity sway velocity and total sway distance using an automatic balance calibration system. Dynamic balance control was assessed using the diagnosis mode of a body-tilt training and measurement system. [Results] Sway velocities on a hard surface with eyes open and closed and those on an unstable sponge surface with eyes closed were significantly higher in the forward head posture group than in the control group. Furthermore, on both the hard and sponge surfaces in the eyes open and closed conditions, total sway distances were significantly higher in the forward head posture group than in the control group. Results of dynamic balance control were not significantly different between groups. [Conclusion] Forward head posture has a greater effect on static balance control than on dynamic balance control.

Key words: Forward head posture, Static balance control, Dynamic balance control
\end{abstract}

(This article was submitted Aug. 25, 2015, and was accepted Oct. 21, 2015)

\section{INTRODUCTION}

In forward head posture (FHP), the head protrudes forward from the sagittal plane and appears to be positioned in front of the body, and this condition is considered the most common postural deformity ${ }^{1,2)}$. FHP increases extension of the atlanto-occipital joint and the upper cervical vertebrae as well as flexion of the lower cervical and upper thoracic vertebrae. Furthermore, this posture causes persistent and abnormal contraction of the suboccipital, neck, and shoulder muscles. In FHP, the center of gravity (COG) of the head shifts in the anterosuperior direction, increasing the load on the neck, which causes dysfunction of the musculoskeletal, neuronal, and vascular systems ${ }^{3}$. Additionally, the muscles around the head and shoulders, including the trapezius, sternocleidomastoid, suboccipital, and temporal, are affected by FHP, which further worsens postural deformity. These changes cause persistent and abnormal pressure in the muscles, fascia, and nerves of the neck and shoulders, and rounding of the shoulders occurs to compensate for this deficit, which in turn, causes a high load on the superior trapezius and levator scapula muscles ${ }^{4}$. All of these changes eventually cause tension neck syndrome. Persistent tension in the head and posterior neck muscles can pathologically mimic tension headache. Additionally, FHP alters the COG of the body that lead to mechanical modifications related to postural control in the torso and every joint. The body attempts to adapt to these changes by altering its balance control mechanisms; these adaptations decrease balance ability while performing different activities and increase the risk of falling and musculoskeletal injury ${ }^{5)}$, and ultimately result in limited body function and a high incidence of various diseases.

Previous studies on FHP have focused on postural change according to specific conditions, such as the use of a smartphone or computer6), or measurement of spinal deformity due to such abnormal posture ${ }^{7)}$; however, few studies have evaluated the effects of FHP on balance control. Therefore, the purpose of the present study was to determine the effects of FHP on static and dynamic balance control. 


\section{SUBJECTS AND METHODS}

The present study included 30 individuals with no history of neuromuscular or spinal disease or surgery in the neck or spinal vertebrae. These individuals had not previously participated in a study similar to the present study. They were sufficiently explained the experimental procedures but were not informed of the purpose before the start of the study. The study was approved by the Research and Ethics Committee of Cheongju University. The craniovertebral angle was measured in all participants, and based on the results, participants were divided into an FHP group (craniovertebral angle $<53^{\circ}, \mathrm{n}=14$ ) and a control group (craniovertebral angle $\geq 53^{\circ}, \mathrm{n}=16$ ) $^{8-10}$ ). A common method of assessing FHP is by observing findings on lateral view images ${ }^{10,11}$, which was adopted in the present study. The base of the camera was set at the height of the participant's shoulder. The tragus of the ear was marked, and a plastic pointer was taped to the skin overlying the spinous process of the $\mathrm{C} 7$ vertebra. Then, the craniovertebral angle - defined as the angle between a horizontal line passing through $\mathrm{C} 7$ and a line extending from the tragus of the ear to $\mathrm{C} 7$ - was measured.

Static balance control was assessed using an automatic balance calibration system (I Balance S; CyberMedic Co., Iksan, Korea). This system measures THE COG, sway velocity of COG, and total sway distance from THE COG using 4 load cells installed with a force plate. Participants stood on the instrument with both feet separated comfortably and both hands placed on the chest. Measurements were taken on a hard surface and an unstable sponge surface with their eyes open and closed ${ }^{12)}$.

Dynamic balance control was assessed using the diagnosis mode of a body-tilt training and measurement system (Spine Balance 3D, CyberMedic Co.). The diagnosis mode can be adjusted by manually introducing a tilt of up to $30^{\circ}$ with a motor at a speed of $2 \% \mathrm{~s}$ in 8 directions, including left-rear-right-right-diagonal, and a stop at $30^{\circ}$ for $5 \mathrm{~s}$, which is the apex for each direction. The screen of the instrument turns black to minimize visual feedback during tilting in the 8 different directions. Furthermore, participants used a 9 -axis inertial measurement unit sensor with $0.1^{\circ}$ resolution, and were instructed to maintain an initial trunk posture during tilting of the footplate. For evaluating the results, each zone from A to E was divided in $2^{\circ}$ intervals from the origin, and then conformity of the truck sensor, system direction, and tilt angle were compared. Participants maintained their postures depending on tilting of the footplate, and when the posture changed by more than $2^{\circ}$, the target was moved from the origin and conformity among the truck sensor, system direction, and tilt angle decreased. Using this procedure, the score varied, depending on elapsed time and zone, from 100 in zone A to 80 in zone B, 60 in zone C, 40 in zone $\mathrm{D}$, and 20 in zone E. Balance posture ratio was expressed by converting elapsed time and score in each zone into percentage. In the current study, balance posture ratio was used to assess trunk maintenance capacity. The score evaluates deviation from the center, with a low score indicating high stability ${ }^{13)}$.

The paired t test was used to compare balance control between the FHP and control groups. All statistical analyses were performed using SPSS version 20.0 for Windows (IBM Corporation, Armonk, NY, USA). A p value of $<0.05$ was considered statistically significant.

\section{RESULTS}

General characteristics of the participants are presented in Table 1. The results of static balance control, including COG sway velocity and total sway distance, and dynamic balance control are presented in Tables 2-4.

On the hard surface, COG sway velocities with the eyes open and closed conditions were significantly higher in the FHP group than in the control group $(\mathrm{p}<0.05)$. On the unstable sponge surface, COG sway velocity with the eyes open was not different between groups; however, COG sway velocity with eyes closed was significantly higher in the FHP group than in the control groups $(\mathrm{p}<0.05)$. Furthermore, on both the hard and sponge surfaces, COG total sway distances with eyes open and closed were significantly higher in the FHP group than in the control group $(p<0.05)$. The results of dynamic balance control, although slightly different, were not significant.

\section{DISCUSSION}

Balance control is evaluated by measuring the time taken to maintain a fixed posture or COG according to postural sway, changes in muscle action potential and joint angle, or motion analysis ${ }^{14)}$. Postural sway measurement is most widely used for evaluating balance control and provides information on sensory changes according to alterations in standing surface or during vision block; this measurement helps determine balance control based on COG sway status of the body ${ }^{15}$. If the COG of the body shift from its normal position, high muscle activity is needed to recover from this unstable position ${ }^{16)}$. Therefore, in conditions of structural imbalance, such as FHP, it is difficult to properly adjust changes in the external environment because the autonomic nervous system has a decreased ability to respond to external stimulation ${ }^{17)}$.

In our study, static balance control was significantly worse in the FHP group than in the control group, except for COG sway velocity on an unstable sponge surface with the eyes open. However, no significant difference was noted in dynamic balance control between groups, possibly because of high overall activity of the postural maintenance muscles during measurement of dynamic balance control.

A previous study reported that FHP does not largely influence static balance control due to the compensatory actions of 
Table 1. General characteristics of the subjects included in the study

\begin{tabular}{lcc}
\hline & FHP Group & Control Group \\
\hline Gender (M/F) & $7 / 8$ & $8 / 8$ \\
Age (years) & $22.1 \pm 1.6$ & $21.6 \pm 1.1$ \\
Height (cm) & $166.1 \pm 8.8$ & $168.4 \pm 8.2$ \\
Weight (kg) & $59.4 \pm 10.7$ & $60.4 \pm 11.6$ \\
BMI & $21.3 \pm 2.4$ & $21.25 \pm 2.4$ \\
\hline
\end{tabular}

Values are expressed as the Mean \pm SD. BMI: body mass index
Table 2. Center of gravity sway velocities of each group under different conditions $(\% / s)$

\begin{tabular}{lcc}
\hline Condition & FHP group & Control group \\
\hline Hard surface - eyes open & $0.06 \pm 0.0^{*}$ & $0.05 \pm 0.0$ \\
Hard surface - eyes closed & $0.23 \pm 0.1^{*}$ & $0.18 \pm 0.0$ \\
Sponge surface - eyes open & $0.27 \pm 0.1$ & $0.23 \pm 0.1$ \\
Sponge surface - eyes closed & $0.44 \pm 0.2^{*}$ & $0.32 \pm 0.1$ \\
\hline
\end{tabular}

Values are expressed as mean $\pm \mathrm{SD}$.

*Significant difference between groups $(\mathrm{p}<0.05)$.

FHP: forward head posture
Table 3. Center of gravity total sway distances of each group under different conditions $(\%)$

\begin{tabular}{llc}
\hline Condition & FHP group & Control group \\
\hline Hard surface - eyes open & $470.2 \pm 111.9^{*}$ & $336.9 \pm 58.0$ \\
Hard surface - eyes closed & $446.9 \pm 110.3^{*}$ & $338.4 \pm 55.8$ \\
Sponge surface - eyes open & $530.9 \pm 127.0^{*}$ & $431.3 \pm 103.6$ \\
Sponge surface - eyes closed & $808.6 \pm 314.5^{*}$ & $559.3 \pm 136.6$ \\
\hline
\end{tabular}

Values are expressed as mean \pm SD.

*Significant difference between groups $(\mathrm{p}<0.05)$.

FHP: forward head posture
Table 4. Dynamic balance in each group (\%)

\begin{tabular}{lcc}
\hline Parameter & FHP group & Control group \\
\hline Balance posture ratio & $45.07 \pm 7.8$ & $42.44 \pm 8.6$ \\
Left & $50.43 \pm 19.1$ & $39.00 \pm 14.5$ \\
Back oblique left & $44.50 \pm 12.4$ & $37.06 \pm 11.6$ \\
Back & $42.00 \pm 15.2$ & $37.25 \pm 7.7$ \\
Back oblique right & $35.79 \pm 7.4$ & $37.94 \pm 9.9$ \\
Right & $49.50 \pm 20.0$ & $44.00 \pm 15.5$ \\
Front oblique right & $35.93 \pm 9.3$ & $40.88 \pm 12.2$ \\
Forward & $49.57 \pm 19.0$ & $49.69 \pm 22.0$ \\
Front oblique left & $36.94 \pm 10.3$ & $41.50 \pm 13.8$ \\
\hline
\end{tabular}

Values are expressed as mean $\pm \mathrm{SD}$

FHP: forward head posture

the body to maintain stability in the standing position ${ }^{18)}$. However, participants in this previous study were children. COG is generally lower in children than in adults, and therefore, postural deformity might have had a low impact on balance control in that study. Another previous study evaluated postural control after inducing FHP in college students with normal posture, and found no problems with postural control ${ }^{19)}$. This result may have been because the participants did not have postural deformity associated with the musculoskeletal system and they were aware of the study purpose. In the present study, a difference was noted in balance control between the FHP and control groups because participants in the FHP group had structural changes in the musculoskeletal system due to FHP. Additionally, in the present study, the difference in static balance control was higher in those with their eyes closed than in those with their eyes open, indicating that vision plays an important role in balance control ${ }^{20)}$.

The present study had some limitations. First, it may be difficult to generalize the findings of the present study to the general population or individuals with a history of disease because the participants were healthy adult men and women. Second, other factors besides FHP that could influence balance control were not assessed. Therefore, further studies are needed to identify the effects of other factors on balance control by investigating various factors that contribute to balance control in participants of various ages and patients with FHP.

In conclusion, FHP has a greater effect on static balance control than on dynamic balance control. Therefore, static balance training may help patients overcome problems associated with FHP. However, further studies are needed to confirm the beneficial effects of static balance training in patients with FHP.

\section{ACKNOWLEDGEMENT}

This study was supported by an academic research fund (a special research project) from the Healthcare Science Research Institute of Cheongju University (academic year 2014-2015).

\section{REFERENCES}

1) Cho CY: Survey of faulty postures and associated factors among Chinese adolescents. J Manipulative Physiol Ther, 2008, 31: 224-229. [Medline] [CrossRef] 
2) Dunleavy K, Goldberg A: Comparison of cervical range of motion in two seated postural conditions in adults 50 or older with cervical pain. J Manual Manip Ther, 2013, 21: 33-39. [Medline] [CrossRef]

3) Harrison DE, Harrison DD, Betz JJ, et al.: Increasing the cervical lordosis with chiropractic biophysics seated combined extension-compression and transverse load cervical traction with cervical manipulation: nonrandomized clinical control trial. J Manipulative Physiol Ther, 2003, 26: 139-151. [Medline] [CrossRef]

4) Cole AK, McGrath ML, Harrington SE, et al.: Scapular bracing and alteration of posture and muscle activity in overhead athletes with poor posture. J Athl Train, 2013, 48: 12-24. [Medline]

5) Lee CM, Jeong EH, Freivalds A: Biomechanical effects of wearing high-heeled shoes. Int J Ind Ergon, 2001, 28: 321-326. [CrossRef]

6) Yoo WG: Comparison of the forward head angle and the lumbar flexion and rotation angles of computer workers using routine and individually fixed computer workstations. J Phys Ther Sci, 2014, 26: 421-422. [Medline] [CrossRef]

7) Gong W: The effects of cervical joint manipulation, based on passive motion analysis, on cervical lordosis, forward head posture, and cervical ROM in university students with abnormal posture of the cervical spine. J Phys Ther Sci, 2015, 27: 1609-1611. [Medline] [CrossRef]

8) Yip CH, Chiu TT, Poon AT: The relationship between head posture and severity and disability of patients with neck pain. Man Ther, 2008, 13: 148-154. [Medline] [CrossRef]

9) Diab AA, Moustafa IM: The efficacy of forward head correction on nerve root function and pain in cervical spondylotic radiculopathy: a randomized trial. Clin Rehabil, 2012, 26: 351-361. [Medline] [CrossRef]

10) Salahzadeh Z, Maroufi N, Ahmadi A, et al.: Assessment of forward head posture in females: observational and photogrammetry methods. J Back Musculoskeletal Rehabil, 2014, 27: 131-139. [Medline]

11) Quek J, Pua YH, Clark RA, et al.: Effects of thoracic kyphosis and forward head posture on cervical range of motion in older adults. Man Ther, 2013, 18: 65-71. [Medline] [CrossRef]

12) Ko MH, Park BH, Chong WS, et al.: Effect of newly developed balance training system in patients with stroke. Appl Mech Mater, 2013, 373-375: 1943-1948. [CrossRef]

13) Shin SH, Yu M, Jeong GY et al.: Effect on the balance ability after four week training using the system for 3-D dynamic exercise equipment. J Rehabil Welf Engineer Assist Techno, 2012, 6: 1-8.

14) Massion J, Amblard B, Assaiante C, et al.: Body orientation and control of coordinated movements in microgravity. Brain Res Brain Res Rev, 1998, 28: 83-91. [Medline] [CrossRef]

15) Kim HS, Lee CW, Lee IS: Comparison between the effects of horseback riding exercise and trunk stability exercise on the balance of normal adults. J Phys Ther Sci, 2014, 26: 1325-1327. [Medline] [CrossRef]

16) Shumway CA, Woolacott MH: American academy of family family phusicians. Mot Contr, 1995, 56: 1815-1823.

17) Ki SH, Song YK: Correlations of forward head posture to heart rate variability and standing posture balance factors. J Korean Med Rehab, 2014, 24: 163-176.

18) Um JY: Correlation between forward head posture and body weight support distribution \& static balance ability of children in growth phase. Kyoung Hee University Graduate School of Physical Education Major in Sports Science and Medicine, 2014.

19) Silva AG, Johnson MI: Does forward head posture affect postural control in human healthy volunteers? Gait Posture, 2013, 38: 352-353. [Medline] [CrossRef]

20) Hytönen M, Pyykkö I, Aalto H, et al.: Postural control and age. Acta Otolaryngol, 1993, 113: 119-122. [Medline] [CrossRef] 